\section{Practical Issues for Quantitative X-ray Microanalysis in SEM at Low kV}

\section{Peter Statham}

Oxford Instruments Analytical Limited, High Wycombe, Bucks U.K. Peter.STATHAM@oxinst.co.uk

In the three decades following Castaing's seminal thesis [1] x-ray analysis received widespread attention from research groups. By 1980, the methods and correction procedures for quantitative analysis of elements with atomic number 11 and above, using accelerating voltages between $15 \mathrm{kV}$ and $25 \mathrm{kV}$, were well established and available in commercial instrumentation. At the time, scanning electron microscopes (SEMs) could rarely deliver high and stable beam current at much lower $\mathrm{kV}$, and $\mathrm{x}$-ray spectrometers had poor efficiency below $1 \mathrm{keV}$ so that low $\mathrm{kV}$ analysis received comparatively little attention. While academic interest in the theory waned, energy dispersive $\mathrm{x}$-ray (EDX) spectrometer technology continued to improve and nowadays most EDX systems include thin polymer window detectors with efficient detection of radiation down to $\mathrm{C}(277 \mathrm{eV})$ and below. Furthermore, the emergence of Schottky field emission SEMs has provided stable low $\mathrm{kV}$ excitation. The widespread availability and ease of use of modern instrumentation might suggest that quantitative microanalysis can be achieved under any set of microscope conditions. However, low $\mathrm{kV}$ analysis introduces a number of problems and proposed solutions have never been subjected to the same extent of peer appraisal that helped refine the state of the art in the early days. This note provides some insight into the difficulties, suitable precautions and methods of validation that can be used to improve the reliability of quantitative results obtained at low $\mathrm{kV}$.

Many materials oxidise when exposed to the atmosphere, so their surface is typically covered by a thin layer of oxide from a few to tens of nm in thickness. Water molecules may be adsorbed and the surface may be covered with hydrocarbon contamination or a conductive carbon coating may be evaporated to reduce specimen charging in the SEM. Consequently, there is often a surface layer rich in $\mathrm{O}$ and C. At $20 \mathrm{kV}$,

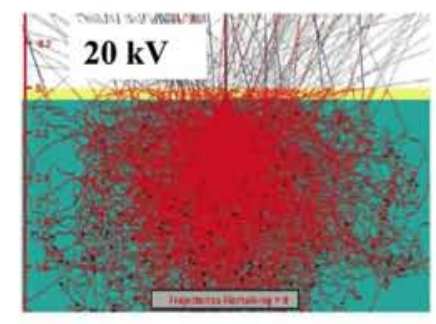

- Small energy loss in layer

- Most X-ray production within sample

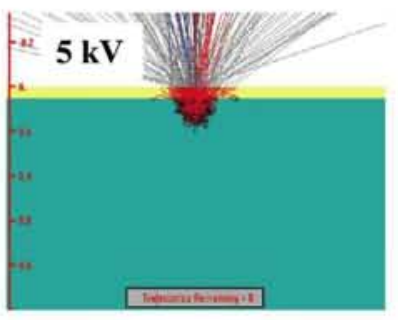

- Significant energy loss in layer

- Relatively large artefact

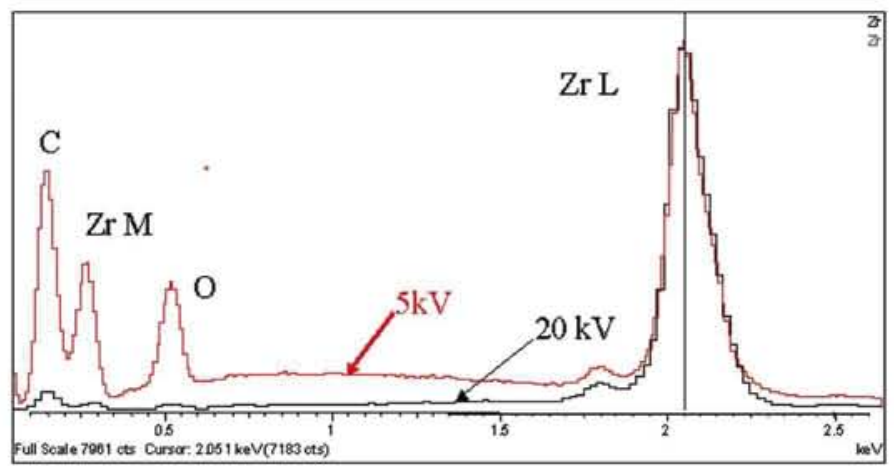

FIG 1: $5 k \mathrm{~V}$ and $20 \mathrm{kV}$ spectra, C-coated $\mathrm{Zr}$ std, scaled at $\mathrm{ZrL}$ incident electrons easily penetrate this layer and the majority of $\mathrm{x}$-ray production occurs in the bulk material below the surface. However, $5 \mathrm{keV}$ electrons are more strongly affected by the layer and a much high proportion of $\mathrm{x}$-ray production occurs in the film. Fig 1 shows the change in an observed spectrum from a carbon coated pure $\mathrm{Zr}$ standard. A large increase in $\mathrm{C} K, \mathrm{O} K$ and $\mathrm{Zr} M$ peaks occurs when the voltage is dropped from $20 \mathrm{kV}$ to $5 \mathrm{kV}$ and the low $\mathrm{kV}$ spectrum clearly does not reflect the true composition of the bulk specimen.

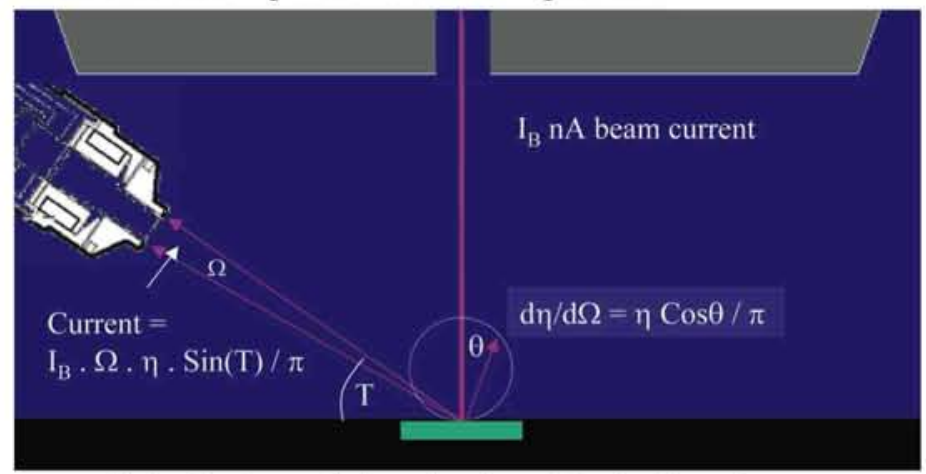

Fig.2 During analysis, a fraction of the incident electrons are backscattered towards the detector.

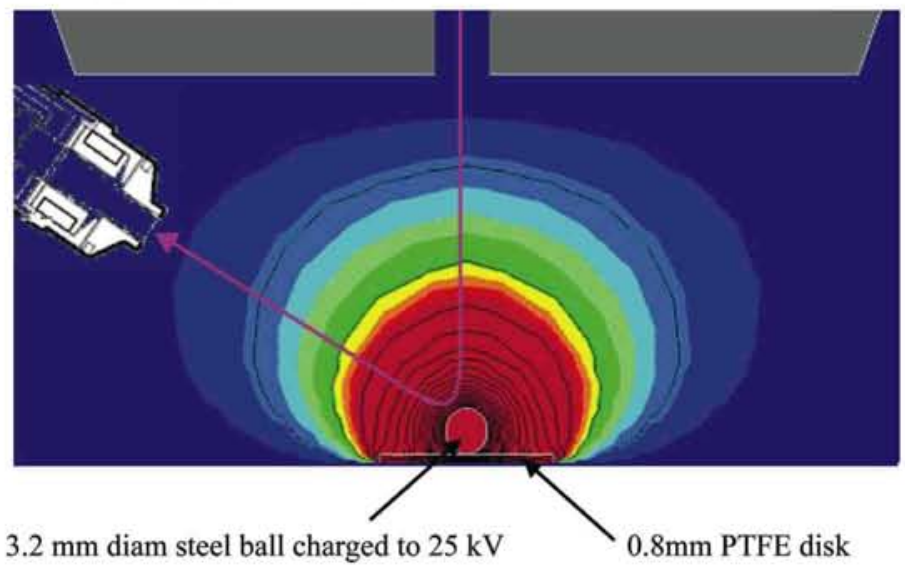

Fig. 3 Trajectory of $5 \mathrm{keV}$ incident electrons when the ball is charged to $25 \mathrm{kV}$.

Corrections for such a layer are possible in principle provided intensities are accurate and any stray radiation contribution eliminated Backscattered electrons provide the most common source of stray radiation in the SEM. Those travelling towards the $\mathrm{x}$-ray detector may excite $\mathrm{C}$ and $\mathrm{O}$ from the polymer window or aquadagged coated surfaces in the collimator unless the electrons are deflected away by the electron trap magnetic field. Even a freshly cleaned specimen may rapidly accumulate oxide and contamination so it is not straightforward to establish whether there are any spurious $\mathrm{O}$ and $\mathrm{C}$ contributions. Therefore, the following method was developed to investigate the level of artefacts. A simplified view of the SEM chamber is shown in fig.2. For an $\mathrm{Al}$ specimen the backscatter coefficient $\eta=0.2$, take-off-angle $T=30 \mathrm{deg}$, and the solid angle for $\mathrm{x}$-ray collection in the detector $\Omega$ $=0.01$ sterad. Therefore, during acquisition, the incident current $I_{B}$ is generating $\mathrm{x}$-rays from the specimen while a fraction of roughly $\left(\eta^{\star} \Omega\right.$ $\left.{ }^{*} \operatorname{Sin}(T) / \pi\right)=0.0003$ is spraying over the collimator area where it may excite spurious $\mathrm{x}$-rays.

An "electron mirror" was constructed by gluing a $3.2 \mathrm{~mm}$ diameter steel ball bearing to a $0.8 \mathrm{~mm}$ thick, $10 \mathrm{~mm}$ diameter clean insulating disk of PTFE, mounted on a standard $\mathrm{Al}$ specimen stub. A spectrum of $\mathrm{Al}$ was acquired at $5 \mathrm{kV}$ from an exposed region on the $\mathrm{Al}$ specimen support stub. The ball was then moved into view and the stage height reduced to focus on the top of the ball. The SEM voltage was 


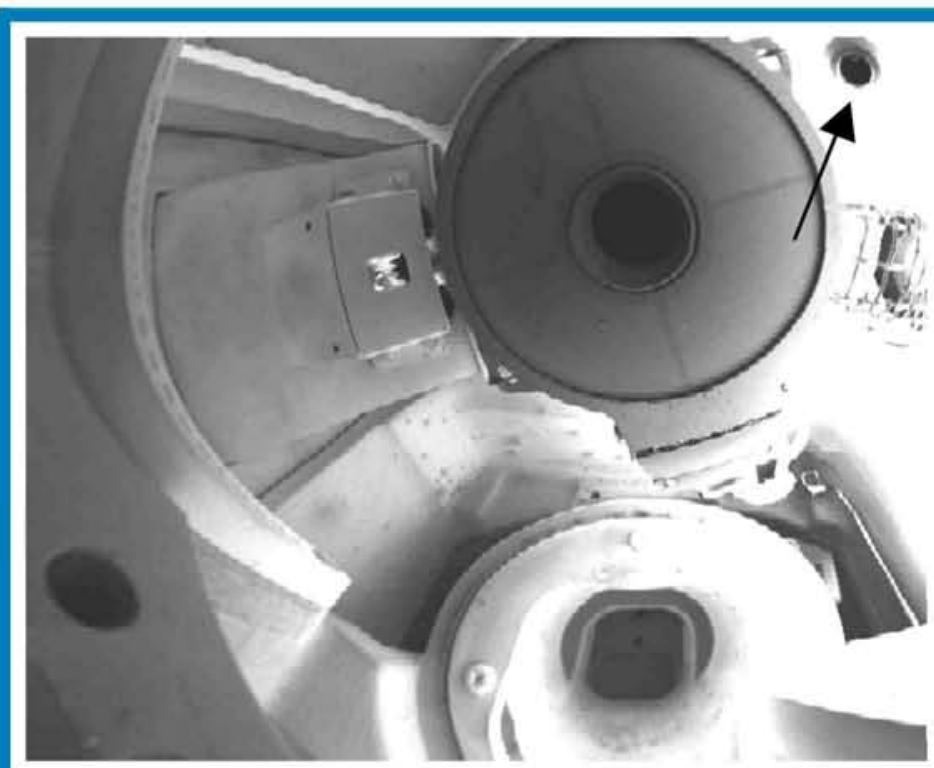

Fig 4: Electron mirror view of inside of SEM chamber.. Arrow shows EDX detector collimator.

then switched to $25 \mathrm{kV}$ for one minute to charge up the ball. When the voltage was switched back to $5 \mathrm{kV}$, fig. 3 shows how incident electrons were reflected away from the charged ball; by rastering over the top of the ball, an image of the inside of the JSM 6400 specimen chamber was obtained for fig. 4 .

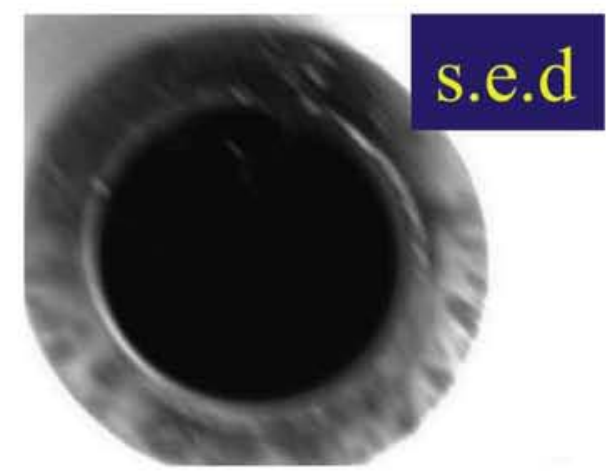

Fig.5 Reduced scan over EDX collimator.

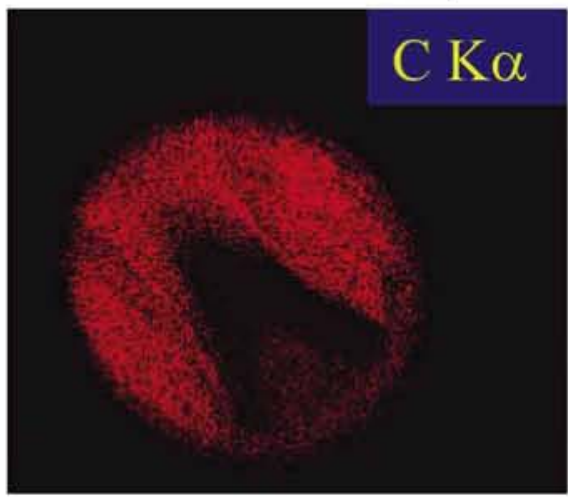

Fig.6 C K $x$-ray map from area in fig. 5
Once artefacts are quantified or eliminated, $x$-ray line intensities from the specimen can be converted to element concentrations using $\mathrm{x}$-ray correction procedures. While suitable algorithms and parameters such as absorption coefficients are available for a wide variety of sample types, accuracy still depends on knowledge of the local geometry and incident beam voltage. At low $\mathrm{kV}$, sample self absorption is reduced and geometry errors are less critical. However, it is often necessary to work at low overvoltages where beam voltage is critical. For example, at $5 \mathrm{kV}$ a change of only $100 \mathrm{eV}$ in beam energy will alter Ca Ka intensity by more than $20 \%$. The SEM voltage cannot be relied upon to be accurate and the most common method of checking the beam voltage is to observe the Duanne Hunt limit (DHL) or the high energy cut-off in the EDX spectrum. If the bremsstrahlung shape conformed to simple "Kramers" theory, then the product of channel count and energy would fall on a straight line. Thus, if this product is fitted to a straight line and extrapolated, the intercept should occur at DHL [2]. In Fig.10, this procedure has been used on a $5 \mathrm{kV}$ spectrum using a $210 \mathrm{eV}$ wide fitting window. The centre of the fitting window is at $4.65 \mathrm{keV}$ and the extrapolated DHL is at $5.02 \mathrm{keV}$. In Fig.11, the lower two curves shows how the product of channel count and energy varies with atomic number of the sample. Whereas low atomic number materials give the expected linear behaviour, high atomic number materials give some curvature to the plot. As a result, the extrapolated DHL changes with the mean energy of the fitting window. For the upper red curve, the extrapolated DHL varies by $200 \mathrm{eV}$ as the fitting window moves over a range of only

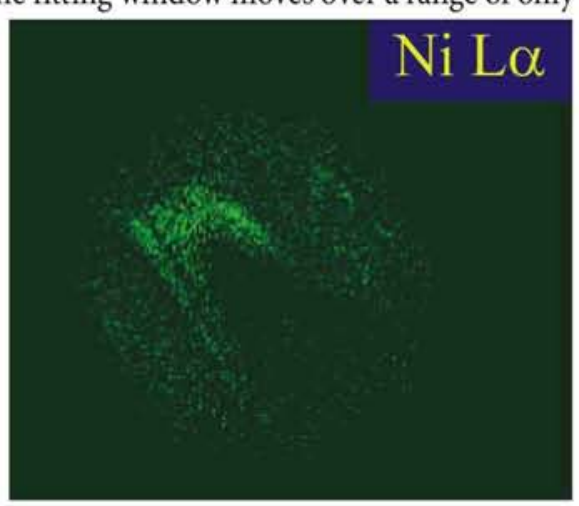

Fig.7 Ni L map from area in fig. 5
A spectrum image ("INCA SmartMap") was recorded using digital beam control with a reduced scan over just the collimator (Fig.5). The $\mathrm{C}$ K map in Fig. 6 shows where electrons hit the carbon-dagged walls of the collimator and the Ni L map, fig.7 shows where electrons end up striking the surfaces of Ni-rich magnets used in the electron trap.

The sum spectrum in Fig. 8 was obtained by integrating over all pixels in the collimator aperture.

Since the full beam current was striking the collimator in "mirror" mode, this spectrum has to be scaled down to see how this spurious contribution would appear when the $\mathrm{Al}$ stub spectrum was recorded. As shown in fig. 2 , only $0.03 \%$ if the incident current falls on the collimator and the sum spectrum has to be scaled by this factor while accounting for any difference in livetime used for obtaining the two spectra. Fig.9. shows the scaled spectra for comparison; the total collimator contribution corresponds to less than $0.2 \%$ of the $\mathrm{Al}$ spectrum and the carbon peak is negligible compared to that from the stub itself. Whereas this may be acceptable for this particular microscope and EDX configuration, the same procedure can be used to qualify any instrument at any $\mathrm{kV}$ to ensure that the collimator and electron trap are effective in eliminating spurious $\mathrm{x}$-rays.
$500 \mathrm{eV}$. The spectra fitted here have exceptional counts and the DHL is normally obscured by statistical scatter. High count rates cannot be used because pile-up contributes counts above DHL and the exact cut off is

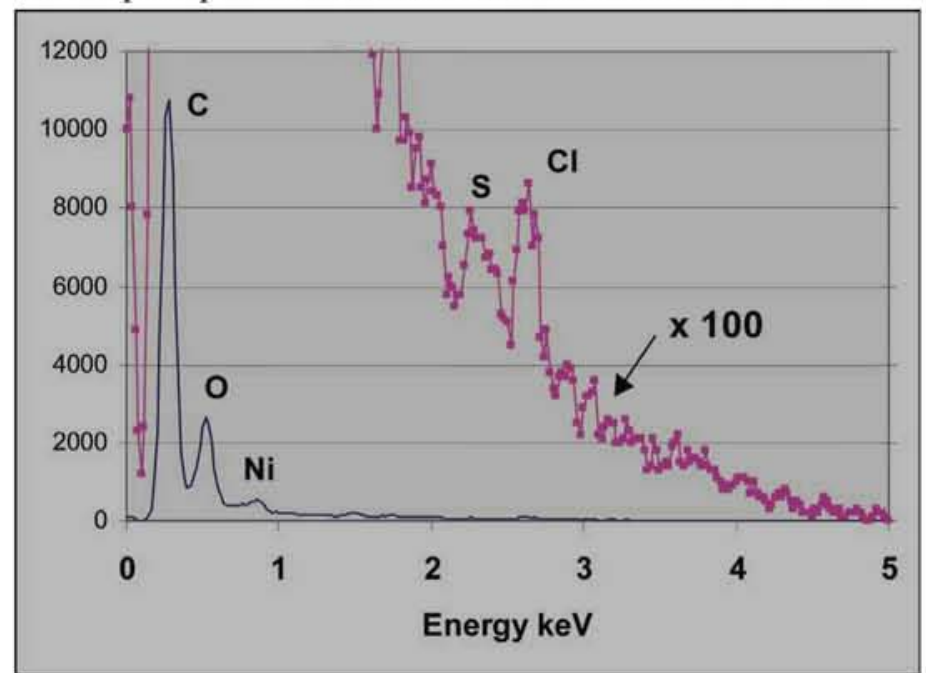

Fig.8 Sum spectrum over all pixels within open area of collimator. Pink curve is scaled by 100 . 


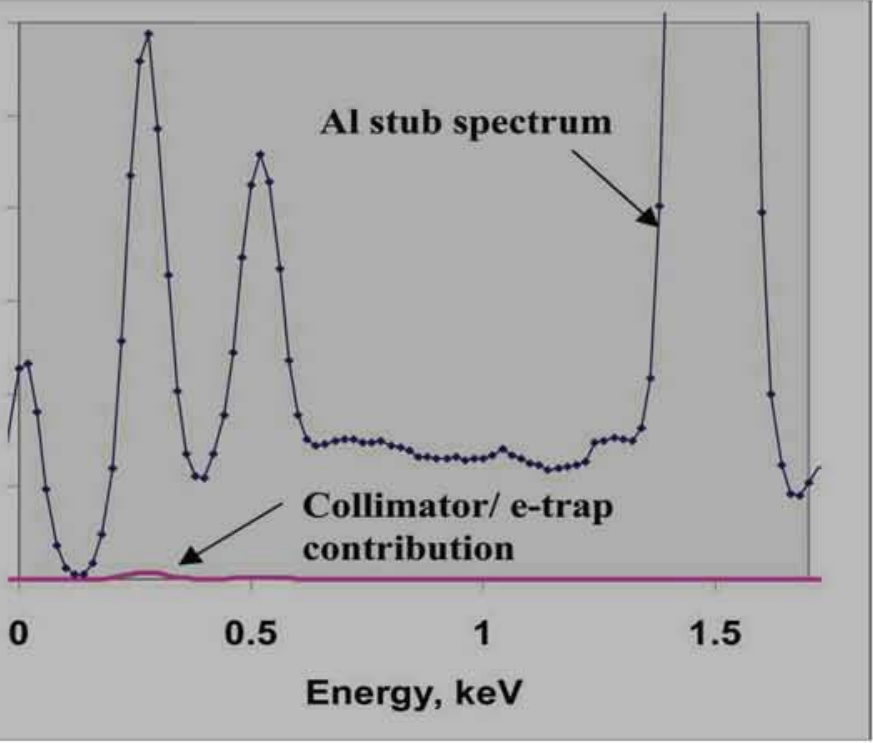

Fig.9 Collimator contribution (pink) for same conditions used for the

$5 \mathrm{kV}$ Al stub spectrum.

smeared out by the detector resolution Therefore, determination of $\mathrm{kV}$ by observation of DHL is not trivial and new methods are required if accuracy better than $100 \mathrm{eV}$ is required.

In addition to the above effects, there may be specimen charging or "skirt" contributions to consider in partial vacuum SEM. Perhaps the greatest source of error is in identification of the elements likely to be present in an unknown sample. At low $\mathrm{kV}$, there are fewer lines available to establish unambiguous identification of an element so the software may miss elements or assign the wrong element to a peak. In Fig. 12 peaks of $\mathrm{As}, \mathrm{Al}$ and $\mathrm{K}$ have been fitted to a $5 \mathrm{kV}$ spectrum

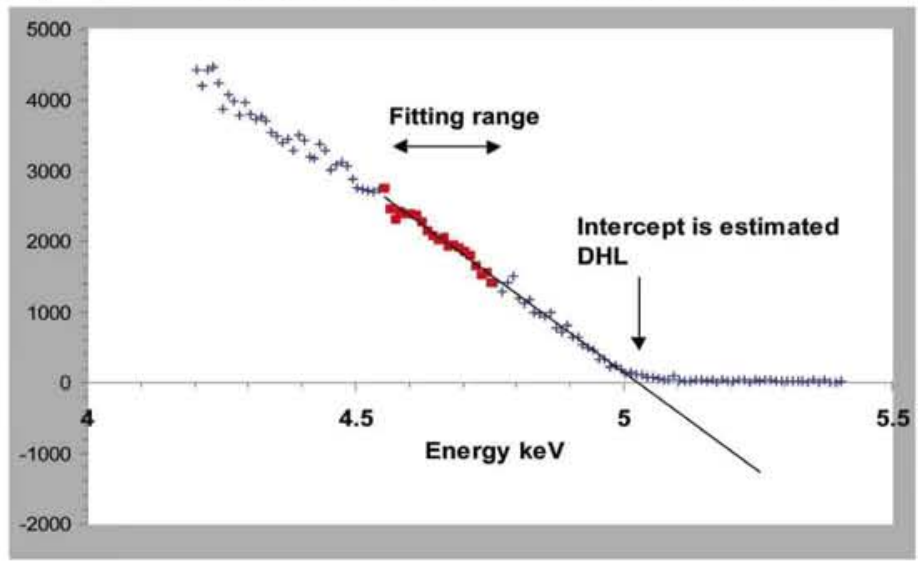

Fig. 10 Extrapolation method for predicting DHL.

Given the possibility that there could be a slight miscalibration and linearity or detector charge collection effects influencing peak shape, the overlay of the fitted result against the yellow histogram bars does not immediately suggest a problem with element identification. The conventional quantitative test that the unnormalised element concentration "Analysis Total" should be close to $100 \%$ demands beam current stability and the measurement of at least one standard-is of no use if results have been normalised to a fixed total. However, if an accurate method is available to predict theoretical spectra [3], consistency of the peak and background content in spectra can be used to check out analyses even when results are normalised. A new "Check Total" has been proposed that has properties similar to the conventional Analysis Total and can be used to validate a normalised set of concentrations [4] Check Total is calculated from the ratio of ( total area of peaks / total

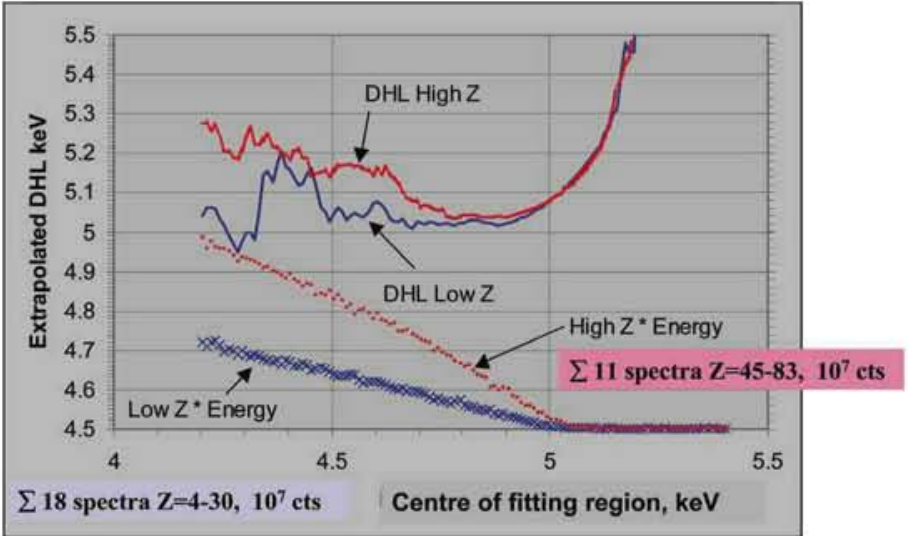

Fig.11 A total of 18 spectra from materials with $Z=4-30$ were summed to give a spectrum with $10^{7}$ counts; channel counts were multiplied by energy to give the blue plot "Low $Z^{\star} E$ nergy". Similarly, 11 spectra from materials with $Z=45-83$ were summed to give a spectrum also with about $10^{7}$ total count for "High $Z^{*}$ Energy" curve in red. As in Fig. 10, a straight line was fitted over a $210 \mathrm{eV}$ energy band and extrapolated to find the DHL. The centre of the energy band used for fitting ( $x$ axis) was varied to see how this affected the extrapolated DHL (y axis) and the results are shown as "DHL Low Z" (blue) and "DHL High Z (red)

background area ) in observed and theoretically simulated spectra. If peak to total background can be predicted with an accuracy of $10 \%$ standard deviation, Check Total is useful for alerting the analyst to incorrect elements, missing peaks, deconvolution errors, and specimen charging [4]. In the Fig. 12 spectrum, when the element intensities were converted to quantitative results, the Check Total value was only $55 \%$ suggesting there was a problem. When the same spectrum was fitted using $\mathrm{Br} L$ and $\mathrm{K} K$ peaks, the quantitative results gave a Check Total of $94 \%$ confirming that $\mathrm{Br}$ and $\mathrm{K}$ was a much more likely solution than $\mathrm{As}, \mathrm{Al}$ and $\mathrm{K}$. In this example, it is the close overlap of $\mathrm{Al} \mathrm{K}$ and $\mathrm{Br} L$,

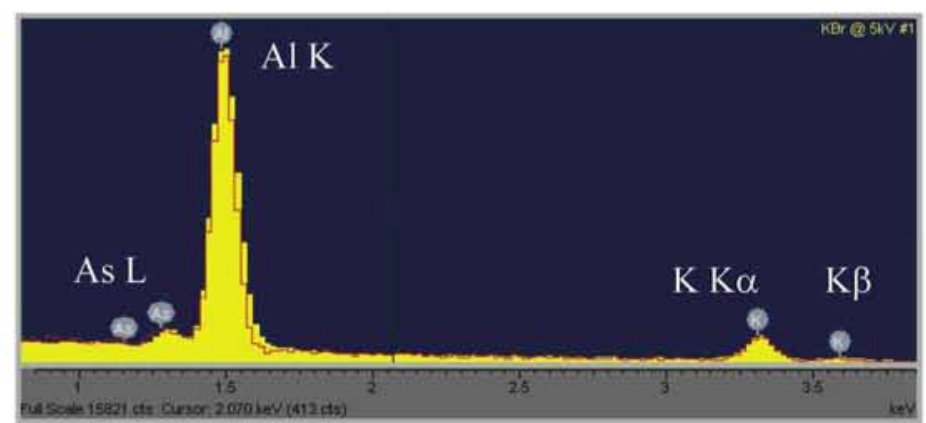

Fig.12 $5 \mathrm{kV}$ spectrum (yellow) showing fit to peaks from As, Al and $K$ (red line).

which are only $7 \mathrm{eV}$ apart, that causes the difficulty in automatic peak identification.

SEM EDX at low $\mathrm{kV}$ offers the exciting prospect of elemental analysis at high spatial resolution. However, the practical issues of surface films, instrumental artefacts and accurate measurement of $\mathrm{kV}$ need to be addressed before reliable quantitative results can be obtained. Some errors are concealed when results are normalised and methods of checking for analysis validity, such as "Check Total," are helpful in preventing serious errors when interpreting x-ray data.

\section{References}

[1]. R. Castaing, Thesis, Univ. of Paris, Paris, France (1951)

[2] R.L.Myklebust, C.E.Fiori and D.E.Newbury, Microbeam Analysis-1990 (1990), 147

[3] Duncumb and P.J. Statham, "Benefits of $\mathrm{x}$-ray spectrum simulation at low energies", Microchimica Acta, 2002, 138, 249-258

[4] P.J.Statham "A check total for validating standardless and normalised edx analysis at low kV" Microchimica Acta, 145, 229-235 (2004). 


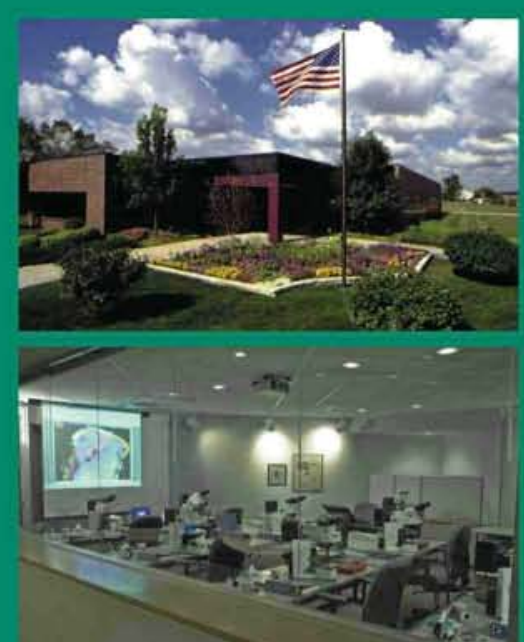

\& COLLEGE OF MICROSCOPY

Located in Westmont, Illinois, the College of Microscopy is an institution for specialized instruction and education, whose goal is to advance the knowledge and understanding of light and electron microscopy for materials analysis.

One of the advantages of attending a course at the College of Microscopy is that our courses are taught primarily by experienced senior staff scientists from McCrone Associates, Inc. who solve micro-analytical problems on a daily basis. That's why it's true when we say,

\section{"Learn From Experience...}

\section{Learn From The Experts."}

\section{Polarized Light Microscopy}

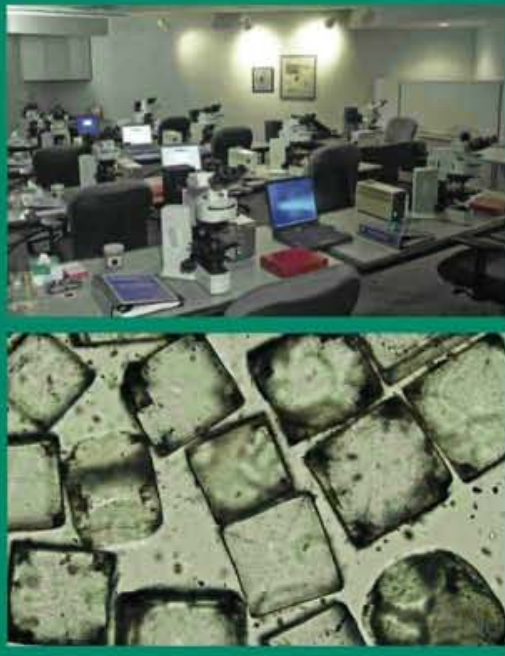

COM100: Polarized Light and Chemical Microscopy

April 3-7, 2006

COM160: Techniques of Optical Crystallography July 17-21, 2006

\section{Electron Microscopy}

COM200: Scanning Electron Microscopy

March 27-31, 2006

COM240: Advanced Electron Microprobe Training Taught by Peter McSwiggen, Ph.D.

Oct. 2-6, 2006

COM250: Transmission Electron Microscopy

Oct. 31-Nov. 2, 2006

\section{Imaging/Software}

COM500: Image Analysis Workshop

April 25-27, 2006

Taught by John Russ, Ph.D.

\section{Sample Preparation}

COM300: Microscopic Particle Handling

COM310: Pharmaceutical and Medical Devices

COM311: Polymers, Paint, and Coatings

COM312: Forensics and Trace Evidence

COM313: Microelectronic Devices and Materials

April 24-28, 2006 July 12-13, 2006 Feb. 22-23, 2006 March 8-9, 2006 April 11-12, 2006

\section{Special Applications}

COM400: Microscopical Examination of Forensic Trace

Aug. 21-25, 2006 Evidence Particles (Part 1)

COM410: Microscopical Identification of Pharmaceutical

July 24-28, 2006 Materials and Contaminants

COM430: Microscopical Identification of WhitePowder Unknowns (Part 1)

May 8-12, 2006

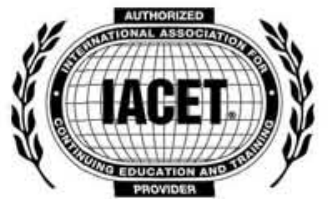

IACET CEUs awarded for all courses completed with the College of Microscopy

College of Microscopy $\bullet 850$ Pasquinelli Drive, Westmont, Illinois 60559 www.collegeofmicroscopy.com 